\title{
Solubility of 3,5-Dinitrosalicylic Acid in Fourteen Pure Solvents over Temperatures from 278.15 to $323.15 \mathrm{~K}$
}

\author{
Xi Zhao, Gui Han, Hongkun Zhao*
}

College of Chemistry \& Chemical Engineering, YangZhou University, YangZhou, Jiangsu 225002, People's

Republic of China

*E-mail address: hkzhao@yzu.edu.cn (H.K. Zhao). 


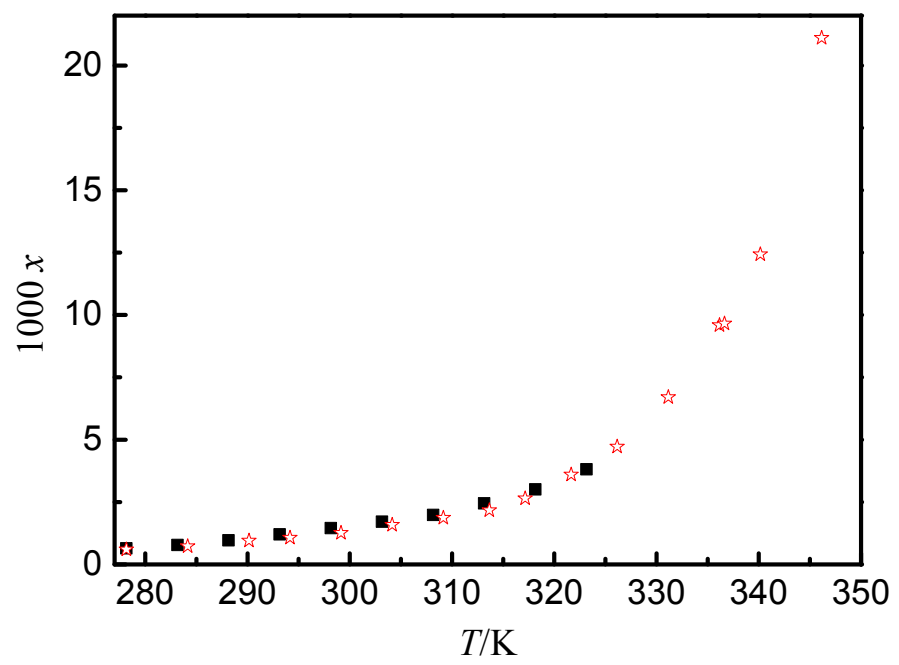

Fig. S1. Solubility of 3,5-dinitrosalicylic acid in water determined in this work and reported in the literature: (a):

口, this work; $ぇ$, Ref. (S1). 


\section{TABLE S1}

The computed values for $\Delta_{\text {mix }} G, \Delta_{\text {mix }} H, \Delta_{\text {mix }} S, \ln \gamma_{1}^{\infty}$ and $H_{1}^{E, \infty}$.

\begin{tabular}{|c|c|c|c|c|c|}
\hline \multirow{2}{*}{$T / \mathrm{K}$} & $\Delta_{\operatorname{mix}} G$ & $\Delta_{\text {mix }} H$ & $\Delta_{\text {mix }} S$ & \multirow{2}{*}{$\ln \gamma_{1}^{\infty}$} & $H_{1}^{E, \infty}$ \\
\hline & $\mathrm{J} \cdot \mathrm{mol}^{-1}$ & $\mathrm{~J} \cdot \mathrm{mol}^{-1}$ & $\mathrm{~J} \cdot \mathrm{mol}^{-1} \cdot \mathrm{K}^{-1}$ & & $\mathrm{~kJ} \cdot \mathrm{mol}^{-1}$ \\
\hline \multicolumn{6}{|c|}{ ethanol } \\
\hline 278.15 & -747.1 & -323.7 & 1.522 & -3.173 & \multirow{10}{*}{-7.535} \\
\hline 283.15 & -823.3 & -354.6 & 1.655 & -3.115 & \\
\hline 288.15 & -913.1 & -391.4 & 1.811 & -3.058 & \\
\hline 293.15 & -1028 & -434.5 & 1.990 & -3.004 & \\
\hline 298.15 & -1143 & -486.6 & 2.202 & -2.952 & \\
\hline 303.15 & -1277 & -541.8 & 2.424 & -2.902 & \\
\hline 308.15 & -1414 & -598.0 & 2.649 & -2.854 & \\
\hline 313.15 & -1553 & -653.6 & 2.871 & -2.807 & \\
\hline 318.15 & -1708 & -716.5 & 3.118 & -2.763 & \\
\hline 323.15 & -1875 & -783.6 & 3.378 & -2.719 & \\
\hline \multicolumn{6}{|c|}{$n$-propanol } \\
\hline 278.15 & -586.1 & -317.1 & 0.967 & -2.950 & \multirow{10}{*}{-9.423} \\
\hline 283.15 & -642.8 & -341.6 & 1.064 & -2.874 & \\
\hline 288.15 & -716.0 & -374.0 & 1.187 & -2.802 & \\
\hline 293.15 & -802.8 & -412.3 & 1.332 & -2.733 & \\
\hline 298.15 & -900.1 & -454.4 & 1.495 & -2.668 & \\
\hline 303.15 & -1003 & -497.4 & 1.667 & -2.605 & \\
\hline 308.15 & -1109 & -540.2 & 1.847 & -2.546 & \\
\hline 313.15 & -1221 & -583.4 & 2.036 & -2.489 & \\
\hline 318.15 & -1338 & -627.2 & 2.235 & -2.434 & \\
\hline 323.15 & -1481 & -679.7 & 2.478 & -2.382 & \\
\hline \multicolumn{6}{|c|}{ isopropanol } \\
\hline 278.15 & -418.1 & -207.8 & 0.756 & -2.451 & \multirow{8}{*}{-8.225} \\
\hline 283.15 & -471.7 & -230.4 & 0.852 & -2.384 & \\
\hline 288.15 & -538.1 & -258.7 & 0.970 & -2.320 & \\
\hline 293.15 & -609.1 & -287.9 & 1.096 & -2.260 & \\
\hline 298.15 & -688.7 & -320.1 & 1.237 & -2.203 & \\
\hline 303.15 & -772.7 & -352.8 & 1.385 & -2.149 & \\
\hline 308.15 & -865.9 & -388.2 & 1.551 & -2.097 & \\
\hline 313.15 & -974.3 & -428.6 & 1.743 & -2.047 & \\
\hline
\end{tabular}




\begin{tabular}{|c|c|c|c|c|c|}
\hline 318.15 & -1091 & -470.4 & 1.950 & -2.000 & \multirow{13}{*}{-6.327} \\
\hline 323.15 & -1231 & -519.7 & 2.203 & -1.955 & \\
\hline \multicolumn{5}{|c|}{ n-butanol } & \\
\hline 278.15 & -182.6 & -78.32 & 0.375 & -1.410 & \\
\hline 283.15 & -218.2 & -92.23 & 0.445 & -1.356 & \\
\hline 288.15 & -260.2 & -108.5 & 0.527 & -1.306 & \\
\hline 293.15 & -295.9 & -121.0 & 0.597 & -1.259 & \\
\hline 298.15 & -348.9 & -140.7 & 0.698 & -1.215 & \\
\hline 303.15 & -423.7 & -169.2 & 0.840 & -1.173 & \\
\hline 308.15 & -476.3 & -186.6 & 0.940 & -1.134 & \\
\hline 313.15 & -566.4 & -219.1 & 1.109 & -1.097 & \\
\hline 318.15 & -639.6 & -242.9 & 1.247 & -1.061 & \\
\hline 323.15 & -746.3 & -279.1 & 1.446 & -1.028 & \\
\hline \multicolumn{6}{|c|}{ isobutanol } \\
\hline 278.15 & -217.5 & -63.20 & 0.555 & -1.554 & \multirow{10}{*}{-4.515} \\
\hline 283.15 & -249.7 & -72.06 & 0.627 & -1.518 & \\
\hline 288.15 & -300.7 & -86.88 & 0.742 & -1.483 & \\
\hline 293.15 & -362.2 & -104.8 & 0.878 & -1.451 & \\
\hline 298.15 & -427.6 & -123.7 & 1.019 & -1.419 & \\
\hline 303.15 & -506.1 & -146.4 & 1.186 & -1.390 & \\
\hline 308.15 & -602.3 & -174.5 & 1.388 & -1.361 & \\
\hline 313.15 & -681.3 & -196.2 & 1.549 & -1.333 & \\
\hline 318.15 & -774.4 & -222.0 & 1.737 & -1.307 & \\
\hline 323.15 & -904.8 & -258.8 & 1.999 & -1.282 & \\
\hline \multicolumn{6}{|c|}{ 1,4-dioxane } \\
\hline 288.15 & -689.2 & -450.8 & 0.828 & -2.809 & \multirow{8}{*}{-11.72} \\
\hline 293.15 & -761.5 & -485.6 & 0.941 & -2.720 & \\
\hline 298.15 & -835.9 & -519.8 & 1.060 & -2.636 & \\
\hline 303.15 & -919.5 & -557.4 & 1.195 & -2.557 & \\
\hline 308.15 & -1006 & -594.5 & 1.337 & -2.482 & \\
\hline 313.15 & -1101 & -633.6 & 1.493 & -2.410 & \\
\hline 318.15 & -1210 & -677.3 & 1.673 & -2.343 & \\
\hline 323.15 & -1331 & -724.2 & 1.877 & -2.279 & \\
\hline \multicolumn{6}{|c|}{ acetonitrile } \\
\hline 278.15 & -166.3 & -73.51 & 0.334 & -1.135 & \multirow{5}{*}{-6.807} \\
\hline 283.15 & -190.0 & -85.04 & 0.371 & -1.083 & \\
\hline 288.15 & -222.1 & -101.1 & 0.420 & -1.033 & \\
\hline 293.15 & -258.8 & -120.1 & 0.473 & -0.984 & \\
\hline 298.15 & -299.4 & -141.6 & 0.530 & -0.937 & \\
\hline
\end{tabular}




\begin{tabular}{|c|c|c|c|c|c|}
\hline 303.15 & -347.4 & -167.8 & 0.593 & -0.892 & \\
\hline 308.15 & -398.3 & -196.3 & 0.656 & -0.848 & \\
\hline 313.15 & -466.3 & -236.1 & 0.735 & -0.806 & \\
\hline 318.15 & -534.8 & -277.5 & 0.809 & -0.765 & \\
\hline 323.15 & -613.8 & -327.1 & 0.887 & -0.725 & \\
\hline & & & etate & & \multirow{11}{*}{-12.97} \\
\hline 278.15 & -752.5 & -537.0 & 0.775 & -3.423 & \\
\hline 283.15 & -809.6 & -564.2 & 0.867 & -3.316 & \\
\hline 288.15 & -880.7 & -599.6 & 0.976 & -3.215 & \\
\hline 293.15 & -965.6 & -642.3 & 1.103 & -3.119 & \\
\hline 298.15 & -1052 & -683.8 & 1.235 & -3.029 & \\
\hline 303.15 & -1156 & -733.7 & 1.392 & -2.943 & \\
\hline 308.15 & -1256 & -780.5 & 1.552 & -2.862 & \\
\hline 313.15 & -1380 & -835.1 & 1.740 & -2.785 & \\
\hline 318.15 & -1510 & -891.4 & 1.945 & -2.711 & \\
\hline 323.15 & -1649 & -948.5 & 2.167 & -2.641 & \\
\hline \multicolumn{6}{|c|}{ EG } \\
\hline 278.15 & -386.2 & -194.7 & 0.688 & -2.277 & \multirow{10}{*}{-8.010} \\
\hline 283.15 & -430.9 & -212.4 & 0.772 & -2.211 & \\
\hline 288.15 & -484.9 & -233.9 & 0.871 & -2.148 & \\
\hline 293.15 & -549.5 & -259.6 & 0.989 & -2.089 & \\
\hline 298.15 & -614.6 & -284.0 & 1.109 & -2.033 & \\
\hline 303.15 & -691.6 & -312.7 & 1.250 & -1.980 & \\
\hline 308.15 & -769.6 & -340.1 & 1.394 & -1.930 & \\
\hline 313.15 & -863.6 & -372.9 & 1.567 & -1.883 & \\
\hline 318.15 & -964.2 & -406.4 & 1.753 & -1.837 & \\
\hline 323.15 & -1082 & -444.8 & 1.973 & -1.794 & \\
\hline \multicolumn{6}{|c|}{ cyclohexane } \\
\hline 283.15 & -44.32 & -4.739 & 0.140 & 0.387 & \multirow{9}{*}{-1.627} \\
\hline 288.15 & -53.69 & -5.841 & 0.166 & 0.399 & \\
\hline 293.15 & -65.58 & -7.281 & 0.199 & 0.411 & \\
\hline 298.15 & -79.72 & -9.042 & 0.237 & 0.422 & \\
\hline 303.15 & -95.89 & -11.11 & 0.280 & 0.433 & \\
\hline 308.15 & -114.3 & -13.53 & 0.327 & 0.443 & \\
\hline 313.15 & -137.9 & -16.74 & 0.387 & 0.453 & \\
\hline 318.15 & -165.2 & -20.60 & 0.455 & 0.463 & \\
\hline 323.15 & -196.6 & -25.19 & 0.530 & 0.473 & \\
\hline \multicolumn{6}{|c|}{ DMF } \\
\hline 278.15 & -1362 & -711.0 & 2.339 & -3.726 & \multirow{2}{*}{-8.675} \\
\hline 283.15 & -1500 & -787.9 & 2.515 & -3.659 & \\
\hline
\end{tabular}




\begin{tabular}{|c|c|c|c|c|c|}
\hline 288.15 & -1658 & -877.4 & 2.709 & -3.595 & \\
\hline 293.15 & -1824 & -973.3 & 2.904 & -3.533 & \\
\hline 298.15 & -2017 & -1087 & 3.118 & -3.473 & \\
\hline 303.15 & -2233 & -1219 & 3.345 & -3.416 & \\
\hline 308.15 & -2446 & -1352 & 3.553 & -3.360 & \\
\hline 313.15 & -2661 & -1489 & 3.745 & -3.306 & \\
\hline 318.15 & -2910 & -1655 & 3.947 & -3.254 & \\
\hline 323.15 & -3170 & -1836 & 4.129 & -3.203 & \\
\hline \multicolumn{6}{|c|}{ NMP } \\
\hline 278.15 & -1471 & -806.1 & 2.390 & -3.769 & \multirow{10}{*}{-9.068} \\
\hline 283.15 & -1624 & -895.2 & 2.573 & -3.699 & \\
\hline 288.15 & -1797 & -998.3 & 2.772 & -3.632 & \\
\hline 293.15 & -1976 & -1106 & 2.967 & -3.567 & \\
\hline 298.15 & -2185 & -1236 & 3.183 & -3.505 & \\
\hline 303.15 & -2414 & -1382 & 3.404 & -3.444 & \\
\hline 308.15 & -2642 & -1531 & 3.606 & -3.386 & \\
\hline 313.15 & -2883 & -1693 & 3.801 & -3.330 & \\
\hline 318.15 & -3157 & -1885 & 3.997 & -3.275 & \\
\hline 323.15 & -3436 & -2090 & 4.163 & -3.223 & \\
\hline \multicolumn{6}{|c|}{ DMSO } \\
\hline 293.15 & -1111 & -454.3 & 2.240 & -2.675 & \multirow{7}{*}{-6.146} \\
\hline 298.15 & -1264 & -525.4 & 2.478 & -2.633 & \\
\hline 303.15 & -1424 & -601.7 & 2.714 & -2.592 & \\
\hline 308.15 & -1592 & -683.7 & 2.950 & -2.552 & \\
\hline 313.15 & -1777 & -776.5 & 3.195 & -2.514 & \\
\hline 318.15 & -1982 & -883.5 & 3.452 & -2.477 & \\
\hline 323.15 & -2216 & -1012 & 3.726 & -2.441 & \\
\hline \multicolumn{6}{|c|}{ water } \\
\hline 278.15 & -9.921 & -1.832 & 0.029 & 1.700 & \multirow{10}{*}{-2.830} \\
\hline 283.15 & -11.81 & -2.210 & 0.034 & 1.722 & \\
\hline 288.15 & -14.32 & -2.732 & 0.040 & 1.743 & \\
\hline 293.15 & -17.49 & -3.412 & 0.048 & 1.763 & \\
\hline 298.15 & -20.74 & -4.118 & 0.056 & 1.782 & \\
\hline 303.15 & -24.03 & -4.843 & 0.063 & 1.801 & \\
\hline 308.15 & -27.45 & -5.606 & 0.071 & 1.819 & \\
\hline 313.15 & -32.99 & -6.913 & 0.083 & 1.837 & \\
\hline 318.15 & -39.44 & -8.489 & 0.097 & 1.854 & \\
\hline 323.15 & -48.18 & -10.73 & 0.116 & 1.871 & \\
\hline
\end{tabular}


a $\Delta_{\text {mix }} G, \Delta_{\text {mix }} H$ and $\Delta_{\text {mix }} S$ signify, respectively, the mixing Gibbs free energy, mixing enthalpy, and mixing entropy; $\gamma_{1}^{\infty}$ refers to the activity coefficient under infinitesimal concentration; and $H_{1}^{E, \infty}$ signifies reduced excess enthalpy under infinitesimal concentration. Standard uncertainties $u$ are $u\left(\Delta_{\text {mix }} G\right)=u\left(\Delta_{\text {mix }} H\right)=0.2 \mathrm{~J} \cdot \mathrm{mol}^{-1}$, $u\left(\Delta_{\mathrm{mix}} S\right)=0.002 \mathrm{~J} \cdot \mathrm{mol}^{-1} \cdot \mathrm{K}^{-1}$. 


\section{REFERENCE}

(S1) Apelblat, A.; Manzurola, E. Solubilities of o-Acetylsalicylic, 4-Aminosalicylic, 3,5-Dinitrosalicylic, and $p$-Toluic Acid, and Magnesium-DL-Aspartate in Water from $T=(278$ to 348) K. J. Chem. Thermodyn. 1999, 31, 85-91. 